\title{
Link Quality Estimators for Multi-Hop Mesh Network
}

\author{
Angela Sara Cacciapuoti*, Marcello Caleffi*, Luigi Paura*† and Md. Arafatur Rahman ${ }^{\ddagger}$ \\ ${ }^{*}$ Department of Electrical Engineering and Information Technologies (DIETI) \\ University of Naples Federico II, Italy \\ ${ }^{\dagger}$ Laboratorio Nazionale di Comunicazioni Multimediali (CNIT), Naples, Italy \\ $\ddagger$ Computer System and Software Engineering, University Malaysia Pahang, Malaysia \\ Email:\{angelasara.cacciapuoti, marcello.caleffi,paura\}@unina.it, arafatur@ump.edu.my
}

\begin{abstract}
In this paper, the problem of estimating the link quality in mesh networks has been considered. Such a problem is a major task to develop an efficient network layer, since an accurate knowledge of the link qualities allows routing protocols to efficiently use neighbors as relays for multi-hop communications. In the last years, a number of link-quality aware routing metrics based on the packet delivery ratio have been proposed and analyzed. However, very few works have addressed the problem of correctly estimating the delivery ratio of a wireless link. Therefore, in this paper we resort to a $802.11 b$ mesh network to carry on a performance comparison between two widely adopted delivery ratio estimators, namely the Simple Moving Average and the Exponentially Weighted Moving Average, and a recently proposed one based on the neural network paradigm.

Index Terms-ad hoc, mesh, 802.11, link quality, estimation, test bed, bio-inspired, neural network.
\end{abstract}

\section{INTRODUCTION}

Wireless mesh networks have attracted tremendous attention due to their properties of self-organization and inexpensive deployment. However, their application in the real world requires the development of a network layer able to assure satisfactory throughput performances [1].

The selection of reliably connected neighbors plays a significant role to successfully and efficiently support data transfers. In fact, experimental results clearly show that the estimation of neighbors' link qualities has a substantiative impact on the network throughput in traditional ad-hoc forwarding [2], [3], as well as in opportunistic forwarding [4], [5], [6] and dynamic spectrum [7], [8] techniques.

In the last years, the problem of designing quality aware routing metrics has received great attention and several metrics have been proposed and evaluated [9], [10], [11], [12], [13], [14], [15]. Despite these efforts, very few works have addressed the problem of correctly estimating the delivery ratio of a wireless link. All the cited metrics assess the link quality by evaluating the delivery ratios or the loss rates with simple estimators, namely the Simple Moving Average (SMA) or the Exponentially Weighted Moving Average (EWMA).

This work was partially supported by the Italian government under PON0102425 "SIRIO: Services for wIreless netwoRk Infrastructure beyOnd 3G", and under PON01-00744 "DRIVEIN2: DRIVEr Monitoring: Technologies, Methodologies, and IN-vehicle INnovative systems for a safe and ecocompatible driving".
Moreover, none of the previous works studied the impact of the parameter setting on the accuracy of the estimators.

For these reasons, in this work we carry on a performance comparison of three link quality estimators: the SMA, the EWMA and the Simple Unsupervised Neuron Estimator (SUNE) [16], a recently proposed estimator based on the neural network paradigm. The performance comparison has been accomplished by using the link-level measurements of the MIT Roofnet topology [10] and by considering several performance metrics.

The outline of the paper is the following: Section II presents the considered estimators, whereas in Section III the performance comparison is described. Finally, in the last section conclusions and open problems are drawn.

\section{Delivery Ratio Estimators}

In this section, we present the three estimators considered for the performance comparison.

All the considered estimators try to assess the quality of a wireless link by resorting to probe packets broadcasted with an average period $\tau$ [2]. Thus, we can model the probe reception event at time $n$ by introducing a binary independent variable $x_{i, j}(n) \in\{0,1\}$, which indicates if the node $j$ has received a probe from the node $i\left(x_{i, j}(n)=1\right)$ or not $\left(x_{i, j}(n)=0\right)$.

Since the wireless channel is time-variant, the probability that the node $j$ receives a probe from the node $i$ depends on the time, namely

$$
P\left(x_{i, j}(n)=1\right)=p_{i, j}(n)
$$

and in the following we refer to such a probability as the delivery ratio $d_{i, j}(n)$ of the link $l_{i, j}$.

\section{A. Simple Moving Average}

The Simple Moving Average (SMA) estimates at time $n$ the delivery ratio $d_{i, j}(n)$ as:

$$
\tilde{d}_{i, j}^{s m a}(n)=\sum_{m=0}^{M-1} b_{m} x_{i, j}(n-m)
$$

where $\mathrm{b}(\mathrm{m})$ is the weighting factor and $M$ is the order of the moving average filter. 


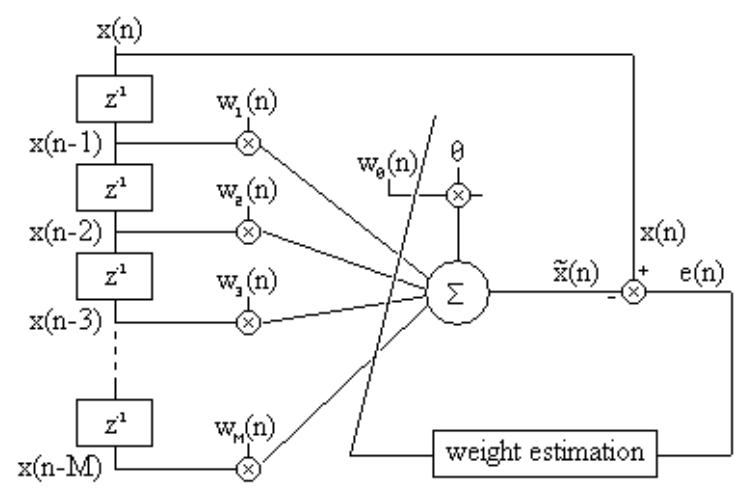

Fig. 1. Simple Un-supervised Neuron Estimator

We note that the SMA requires the setting of both the weighting factors and the order values. Usually [2], the weighting factors are set to the same value $b_{m}=\frac{1}{M} \forall m$ and in such a case the SMA is an un-weighted mean of the previous $M$ realizations of the random variable $x_{i, j}$.

\section{B. Exponential Weighted Moving Average}

The Exponential Weighted Moving Average (EWMA) estimates at time $n$ the delivery ratio $d_{i, j}(n)$ as:

$$
\tilde{d}_{i, j}^{\text {ewma }}(n)=\alpha x_{i, j}(n)+(1-\alpha) \tilde{d}_{i, j}^{\text {ewma }}(n-1)
$$

where $\alpha \in[0,1]$ is the smoothing factor.

We note that the EWMA requires the setting of the only smoothing factor. With respect to the SMA, the EWMA applies to data points weighting factors which decrease exponentially, and the degree of weighting decreasing is expressed by $\alpha$. In other words, the EWMA gives much more importance to recent observations without entirely discarding the older observations.

\section{Simple Unsupervised Neuron Estimator}

The Simple Unsupervised Neuron Estimator (SUNE) [16] is a delivery ratio estimator based on a simple neural network. The processing ability of SUNE is reached by means of connection strengths, or weights, which are set by a process of adaptation, or learning.

The SUNE estimates at time $n$ the delivery ratio $d_{i, j}(n)$ by predicting the next probe reception event $\tilde{x}_{i, j}(n+1)$ basing on the $M$ previous reception events and the bias coefficient $\theta$ as shown in Fig. 1.

More specifically, the estimated delivery ratio is the sum of the $M$ past values, weighted by the coefficients $w_{i}(n) ; 1 \leq$ $i \leq M$, plus bias $\theta$, weighted by the coefficient $w_{\theta}(n)$ :

$$
\tilde{d}_{i, j}^{S U N E}(n)=\sum_{k=0}^{M-1} w_{k}(n-1) x_{i, j}(n-k)+w_{\theta}(n) \theta
$$

To assess the weights $\mathbf{w}(n)=\left[w_{1}(n), \ldots, w_{m}(n), w_{\theta}(n)\right]$ at time $n$, SUNE adopts an unsupervised algorithm that at each

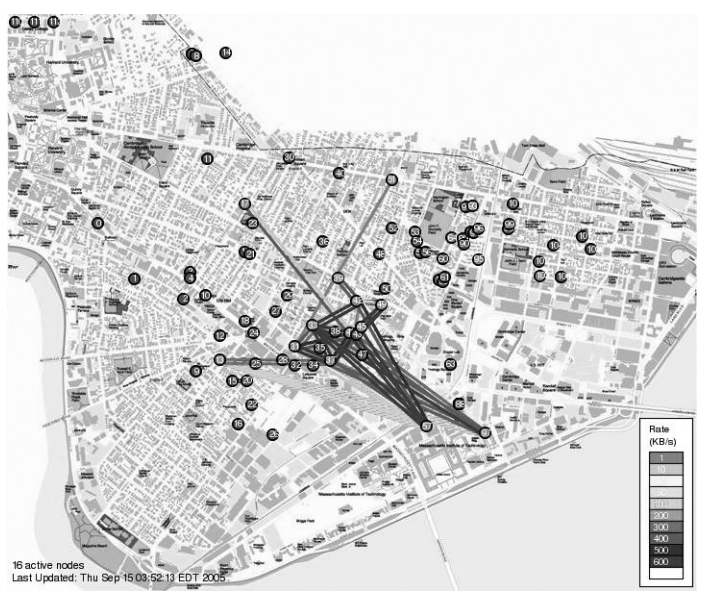

Fig. 2. A map of Roofnet test bed

time should adapts the weight values in order to minimize the estimation error, namely

$$
e_{i, j}^{S U N E}(n)=d_{i, j}(n+1)-\tilde{d}_{i, j}^{S U N E}(n)
$$

Since such an error cannot be evaluated due to the lack of knowledge of the true value $d_{i, j}(m)$, in [16] it is proposed to replace a such an error with the following estimate:

$$
\tilde{e}_{i, j}^{S U N E}(n)=x_{i, j}(n+1)-\tilde{d}_{i, j}^{S U N E}(n)
$$

The minimization criteria is the least square error (LSE) criterion, and the process of estimating the weight values $\mathbf{w}(n)$, namely the learning process, is based on the delta rule [17]:

$$
\begin{gathered}
\Delta \mathbf{w}(n)=2 \eta e_{i, j}(n) \mathbf{x}_{i, j}(n)+\alpha \Delta \mathbf{w}(n) \\
\mathbf{w}(n)=\mathbf{w}(n-1)+\Delta \mathbf{w}(n)
\end{gathered}
$$

where $\mathbf{x}_{i, j}(n)=\left[x_{i, j}(n), \ldots, x_{i, j}(n-M+1)\right], \eta$ is the learning rate and $\alpha$ is the momentum term which controls the speed of convergence.

\section{EXPERIMENTAL RESULTS}

In this section, we present the results of the performance comparison of the three estimators based on a IEEE $802.11 \mathrm{~b}$ test bed.

\section{A. Setup}

To evaluate the performance of the considered estimators, we use the link-level measurements of the MIT Roofnet test bed [10].

The network of the test-bed is composed by 38 nodes IEEE $802.11 b$, and in Fig. 2 shows a map of the network. During the experiment each Roofnet node in turn sends 1500byte packets as fast as possible for 90 seconds at each of the $802.11 \mathrm{~b}$ bitrates: 1, 2, 5.5 and 11Mbps. Meanwhile, all other nodes in the network listen and record in a trace file the received packets. The experiment uses IEEE 802.11 broadcast packets since they involve no link-level acknowledgments or retransmissions. For 
sake of space, in the following we report the results regarding a bit-rate equal to $1 \mathrm{Mbps}$.

We exploit the traces produced by the nodes to generate the probe reception events $x(n) \in\{0,1\}$ used by the estimators to assess the delivery ratios. Moreover, the estimation errors for SMA and EWMA at time $n$ for the link $l_{i, j}$ are respectively:

$$
\begin{aligned}
\tilde{e}_{i, j}^{s m a}(n) & =x_{i, j}(n+1)-d_{i, j}^{s m a}(n) \\
\tilde{e}_{i, j}^{\text {ewma }}(n) & =x_{i, j}(n+1)-d_{i, j}^{\text {ewma }}(n)
\end{aligned}
$$

We note that only links with more than 3000 probe reception events have been considered to improve the accuracy of the statistical estimations and to limit the transitory effects.

As regard to the window size $M$, we study the performances for two values commonly adopted in literature: 10 [2] and 32 [18]. Moreover, we also consider a larger value, say 512, which better accounts for static mesh network topology features like the Roofnet ones.

As regard to the EWMA estimator, we consider two values for the smoothing factor $\alpha$ : 0.1 and 0.9 , referring to the former as slow since in such a case the estimator slowly adapts to the link quality changes and to the latter as fast for the opposite reason.

As regard to the SUNE estimator, we adopt the same setting of the original work [16], namely $\mathbf{w}(0)=\left[\frac{1}{2}, \ldots, \frac{1}{2}, \frac{1}{2}\right], \eta=$ 0.001 and $\alpha=0.5$.

\section{B. Qualitative Comparison}

In this section we present a qualitative performance comparison of the considered estimators by means of density scatter plots.

Each dot $(x, y)$ in the figure represents a probe reception event $x_{i, j}(n)(x)$ and the correspondent probe reception event predicted $\tilde{x}_{i, j}(n)$ by the estimator at time $n-1(y)$. The density of the scatter plot is determined with a 2D histogram, using 25 equally spaced bins in both directions. The more darkness is a zone in the plot, the more dots are present in the same zone. Clearly, for a perfect estimator all the dots would be at the coordinates $(0,0)$ or $(1,1)$.

In the first set of experiments (Fig. 3) we consider a link with a data-rate equal to $1 \mathrm{Mbps}$ and with an average delivery ratio equal to 0.55 . Moreover, the window size $M$ has been set to 10. The results show that the fast EWMA is very sensitive to link quality changes. Therefore, sometimes it is able to correctly estimate the link quality (since we have some darkness zone around $(0,0)$ or $(1,1)$ ) and sometimes it completely screws up (darkness around $(1,0)$ or $(0,1)$ ). The other estimators perform pretty the same with the dots concentrated around $(0,0.5)$ or $(0.5,0)$

In the second set (Fig. 4) we consider the same link of the previous experiments, but we set the window size $M=512$. As regard to the EWMA estimators, their performances are quite similar to those of the previous set, with the slow EWMA outperforming the fast one. On the other hand, the SMA darkness zone is now more concentrated the around $(0,0.5)$ or $(0.5,0)$ while the SUNE performs the best since it presents the darkest zones around $(0,0)$ and $(1,1)$.

\section{Quantitative Comparison}

In this section we present a quantitative performance comparison of the considered estimators for three different values of $M$, namely 10,32 and 500 . For each experiment, we consider two performance metrics: the mean absolute error (MAE)

$$
e_{\text {mae }}=\frac{1}{n} \sum_{k=1}^{n}\left|\tilde{e}_{i, j}(k)\right|
$$

and the mean square error (MSE)

$$
e_{m s e}=\frac{\sum_{k=1}^{n}\left(\tilde{e}_{i, j}(k)\right)^{2}}{n}
$$

In the figure, the $x$ axis represents the value of the average delivery ratio for a certain link, while the $y$ one is the value of the MAE or of the MSE for the same link. We note that the figure presents the results for all the links of the test bed (more than five hundred).

In the first set of experiments (Fig. 5) we plot the MSE and we note that the fast EWMA performs the worst for each value of the window size. As regard to the SUNE, it performs the best for smaller window size, while its performance gain with respect to the SMA and the slow EWMA decreases for the largest window size. Moreover, in such a case its values are notably scattered. Finally, we note that SMA and slow EWMA perform pretty the same.

In the second set (Fig. 6) we plot the MAE. The results substantiate the comments made previously. More in detail, the MAE values of all the estimators are less than the MSE ones due to the effects of the square function. Moreover, with respect to the SUNE performances for $M=512$, we note that performs the worst when the average delivery ratios assume edge values.

\section{Conclusion}

In this paper, the problem of estimating the link quality in mesh networks has been considered. More in detail, by resorting to the link-level measurements of a $802.11 b$ test bed, we carry on a performance comparison between three delivery ratio estimators, namely the the Simple Moving Average (SMA), the Exponentially Weighted Moving Average (EWMA) and the Simple Unsupervised Neuron Estimator (SUNE) for different values of the estimator order. The results of the comparison substantiates that the performances of the estimators depend from several parameters, although novel techniques based on neural network paradigm, like SUNE, seem able to perform well in different environmental conditions.

\section{REFERENCES}

[1] E. Ancillotti, R. Bruno, and M. Conti, "Experimentation and performance evaluation of rate adaptation algorithms in wireless mesh networks," in Proc. of the 5th ACM symposium on Performance evaluation of wireless ad hoc, sensor, and ubiquitous networks (PE-WASUN), 2008, pp. 7-14.

[2] D. S. J. D. Couto, D. Aguayo, J. Bicket, and R. Morris, "A highthroughput path metric for multi-hop wireless routing," Wireless Networks, vol. 11, no. 4, pp. 419-434, 2005. 
[3] R. Draves, J. Padhye, and B. Zill, "Comparison of routing metrics for static multi-hop wireless networks," in Proc. of the ACM Conference on Applications, technologies, architectures, and protocols for computer communications (SIGCOMM), 2004, pp. 133-144.

[4] A. S. Cacciapuoti, M. Caleffi, and L. Paura, "A theoretical model for opportunistic routing in ad hoc networks," in Proc. of IEEE International Conference on Ultra Modern Telecommunications Workshops (ICUMT), 2009, pp. 1-7.

[5] S. Biswas and R. Morris, "Exor: opportunistic multi-hop routing for wireless networks," SIGCOMM Comput. Commun. Rev., vol. 35, no. 4, pp. 133-144, 2005.

[6] A. S. Cacciapuoti, M. Caleffi, and L. Paura, "Optimal constrained candidate selection for opportunistic routing," in Proc. of IEEE Global Telecommunications Conference (GLOBECOM), 2010, pp. 1-5.

[7] A. S. Cacciapuoti, C. Calcagno, M. Caleffi, and L. Paura, "Caodv: Routing in mobile ad-hoc cognitive radio networks," in IEEE IFIP Wireless Days, 2010, pp. 1-5.

[8] A. S. Cacciapuoti, M. Caleffi, and L. Paura, "Reactive routing for mobile cognitive radio ad hoc networks," Ad Hoc Networks (Elsevier), vol. 10, no. 5 , pp. $803-815,2012$.

[9] A. Adya, P. Bahl, J. Padhye, A. Wolman, and L. Zhou, "A multi-radio unification protocol for ieee 802.11 wireless networks," in Proc. of the First International Conference on Broadband Networks (BroadNets), Oct. 2004, pp. 344-354.

[10] D. Aguayo, J. Bicket, S. Biswas, G. Judd, and R. Morris, "Link-level measurements from an 802.11 b mesh network," SIGCOMM Comput. Commun. Rev., vol. 34, no. 4, pp. 121-132, 2004.

[11] R. Draves, J. Padhye, and B. Zill, "Routing in multi-radio, multi-hop wireless mesh networks," in Proc. of the 10th annual international conference on Mobile computing and networking (MobiCom), 2004, pp. 114-128.

[12] K.-H. Kim and K. G. Shin, "On accurate measurement of link quality in multi-hop wireless mesh networks," in Proc. of the 12th annual international conference on Mobile computing and networking (MobiCom), 2006, pp. 38-49.

[13] R. Fonseca, O. Gnawali, K. Jamieson, and P. Levis, "Four-bit wireless link estimation," in Proc. of the Sixth Workshop on Hot Topics in Networks (HotNets VI), Nov 2007.

[14] A. Vlavianos, L. K. Law, I. Broustis, S. V. Krishnamurthy, and M. Faloutsos, "Assessing link quality in ieee 802.11 wireless networks: Which is the right metric?" in Proc. of IEEE 19th International Symposium on Personal, Indoor and Mobile Radio Communications (PIMRC), Sept. 2008, pp. 1-6.

[15] M. Campista, P. Esposito, I. Moraes, L. Costa, O. Duarte, D. Passos, C. de Albuquerque, D. Saade, and M. Rubinstein, "Routing metrics and protocols for wireless mesh networks," Network, IEEE, vol. 22, no. 1, pp. 6-12, Jan.-Feb. 2008.

[16] M. Caleffi and L. Paura, "Bio-inspired link quality estimation for wireless mesh networks," in Proc. of the 10th IEEE International Symposium on a World of Wireless, Mobile and Multimedia Networks (WoWMoM), June 2009.

[17] D. Graupe, Principles of artificial neural networks. World Scientific Publishing Co., Inc., 1997.

[18] H. Rogge, E. Baccelli, and A. Kaplan, "Packet sequence number based etx metric for mobile ad hoc networks," Internet Engineering Task Force (IETF), Internet-Draft, March 2010.
Fig. 3. Density scatter plot for $M=10$ SMA: data-rate $=1 \mathrm{Mbps}$, windows - size $=10$

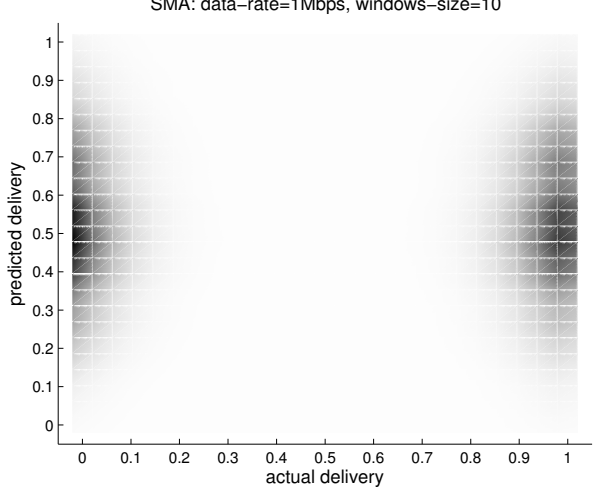

Fast EWMA: data-rate $=1 \mathrm{Mbps}$, windows-size $=10$

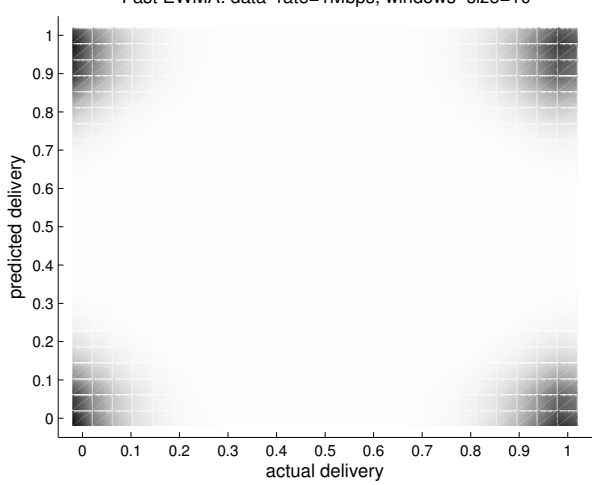

Slow EWMA: data-rate $=1 \mathrm{Mbps}$, windows - size $=10$

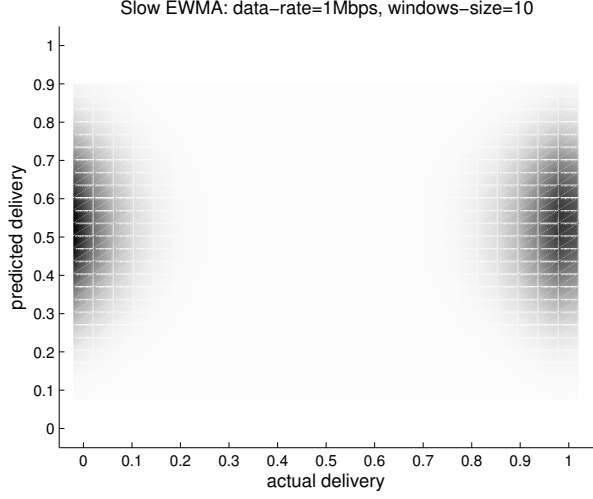

SUNE: data-rate $=1 \mathrm{Mbps}$, windows-size $=10$

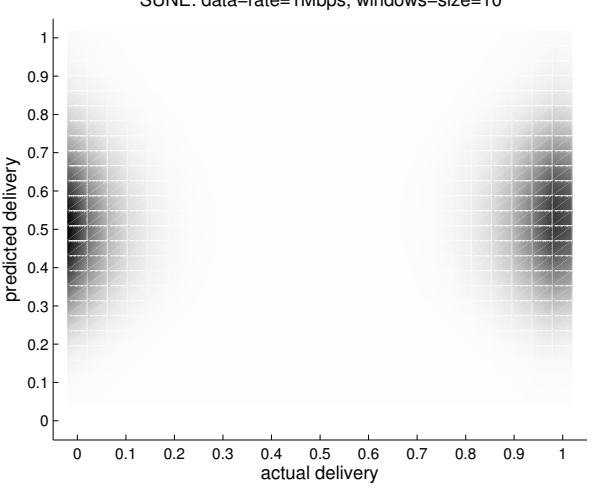


Fig. 4. Density scatter plot for $M=512$ SMA: data-rate $=1 \mathrm{Mbps}$, windows-size $=512$

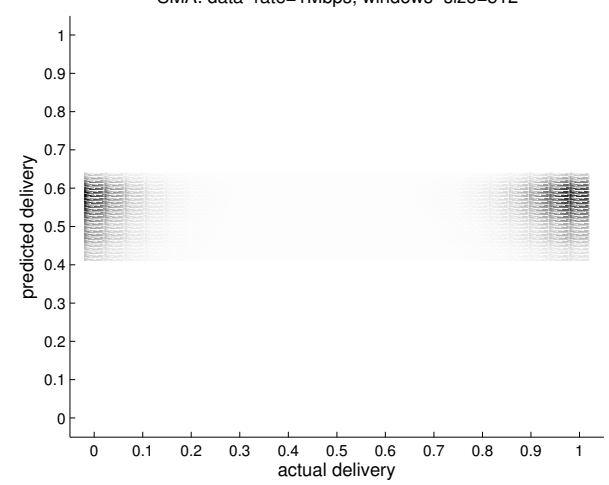

Fast EWMA: data-rate $=1 \mathrm{Mbps}$, windows - size $=512$

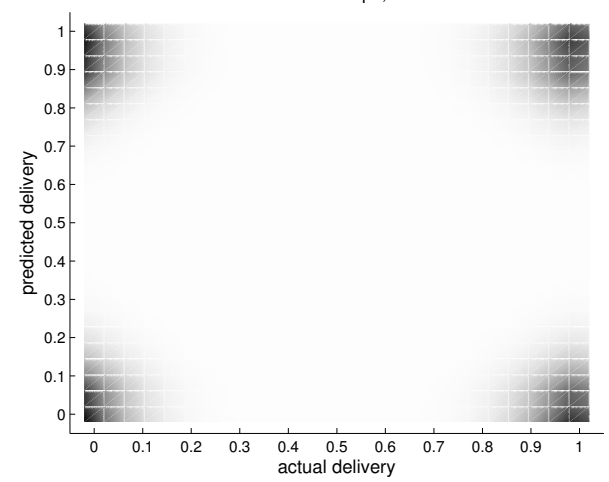

Slow EWMA: data-rate $=1 \mathrm{Mbps}$, windows - size $=512$

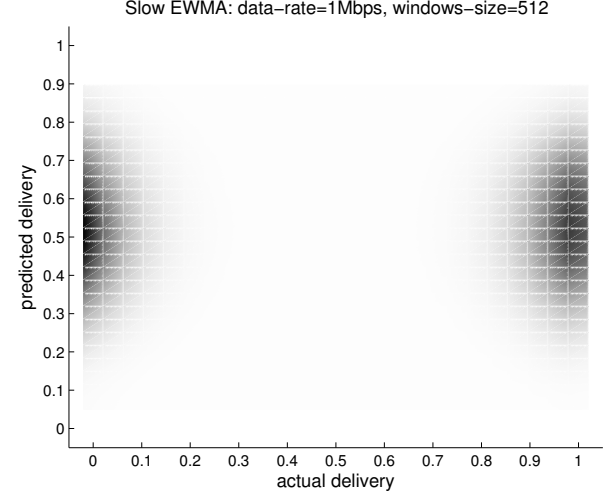

SUNE: data-rate $=1 \mathrm{Mbps}$, windows-size $=512$

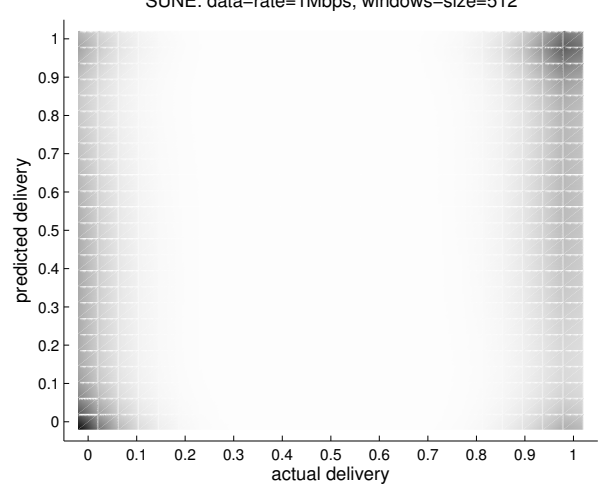

Fig. 5. Mean Square Error
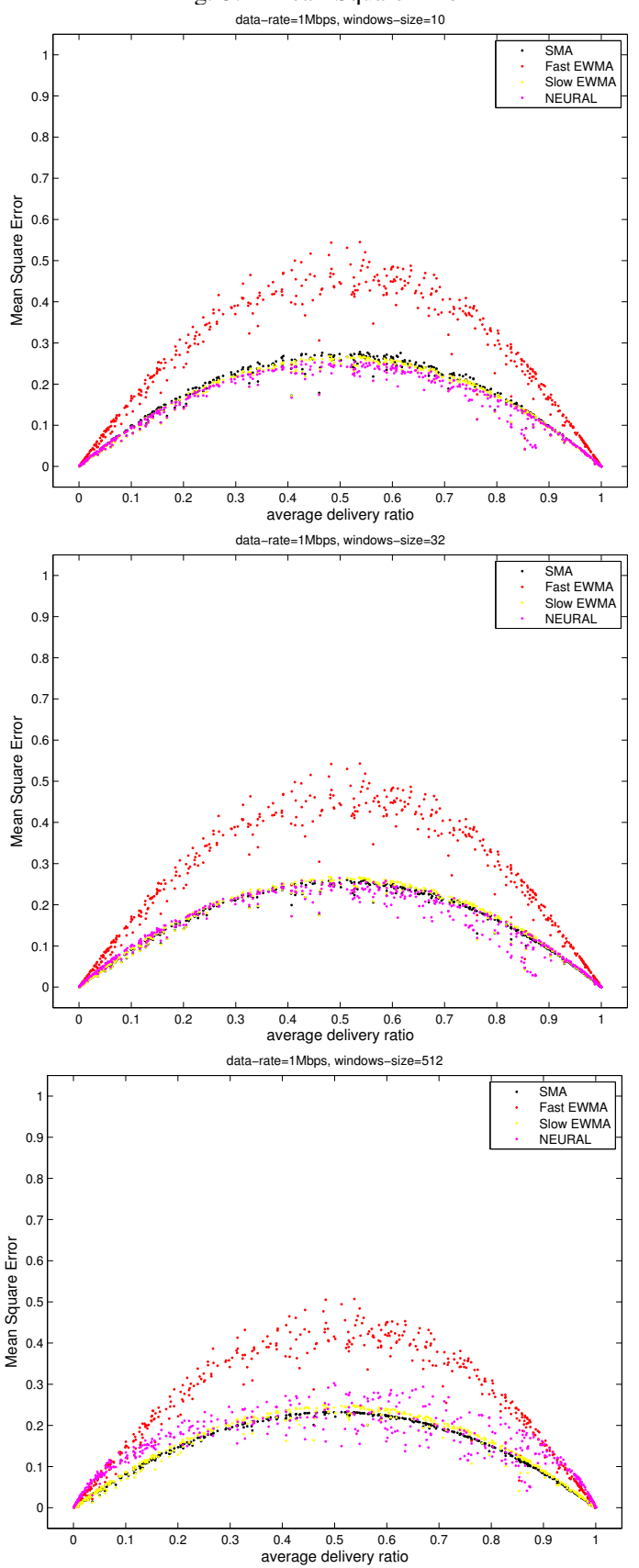
Fig. 6. Mean Absolute Error
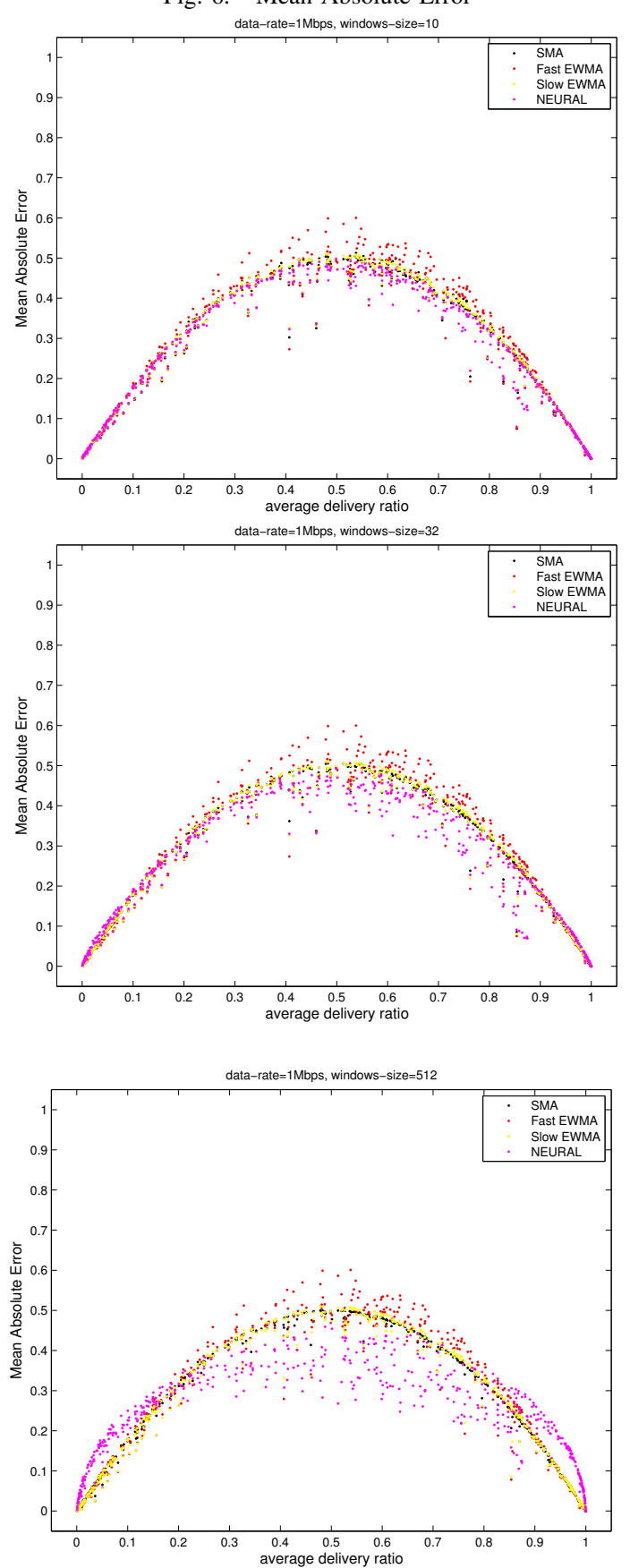